\section{Tödliche Kombination}

oraussetzung für Leistungen aus der gesetzlichen Unfallversicherung ist, dass bestimmte Schadstoffgrenzwerte am Arbeitsplatz überschritten werden und diese Schadstoffbelastung auch ursächlich für die Erkrankung war. Nach der bisherigen Rechtsprechung wurde eine Anerkennung als „Berufskrankheit" abgelehnt, wenn ein bestimmter Schadstoff unterhalb des Grenzwertes lag. Das Hessische Landessozialgericht hat nun erstmals die Ansicht vertreten, dass auch eine berufsbedingte Schadstoffkombination einen Anspruch auf Entschädigung begründen kann. Die Richter gaben der Witwe eines Dachdeckers Recht, der am Arbeitsplatz neben Asbest auch schädlichen Dämpfen von Heißteer und Heißbitumen ausgesetzt war und an Lungenkrebs verstarb:

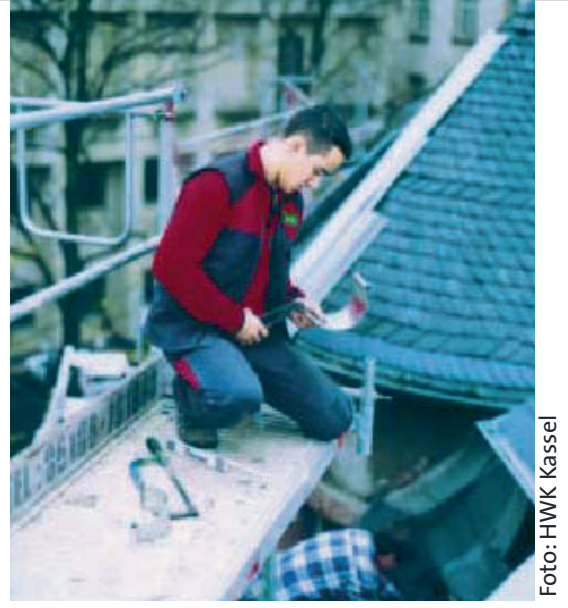

Diese Kombinationsschädigung sei für den Tod ursächlich gewesen, die Witwe habe einen Leistungsanspruch aus der gesetzlichen Unfallversicherung. Dies gelte auch dann, wenn der Grenzwert eines bestimmten Schadstoffes noch nicht überschritten wurde.

$j l p$

Hessisches Landessozialgericht, Az. L 11/3

U 740/02 ZVW

\title{
Sinnlose Keimkiller in Reinigungsmitteln
}

einigungsmittelhersteller bewerben ihre Produkte gern als ,antibakteriell wirksam". Vielleicht sollte man dies den Krankheitserregern auch mitteilen: Eine Doppelblindstudie in 238 Haushalten, in denen mindestens ein Vorschulkind lebte, ergab nämlich, dass die Kinder in der aspetischen Umwelt genau so häufig an Infektionserkrankungen litten wie Kinder in Hauhalten, in denen keine antibakteriellen Putzmittel verwendet wurden. $D E$

Larson EL et al. Ann Intern Med 2004; 140: 321-9

\section{Phototoxischer Tabakqualm}

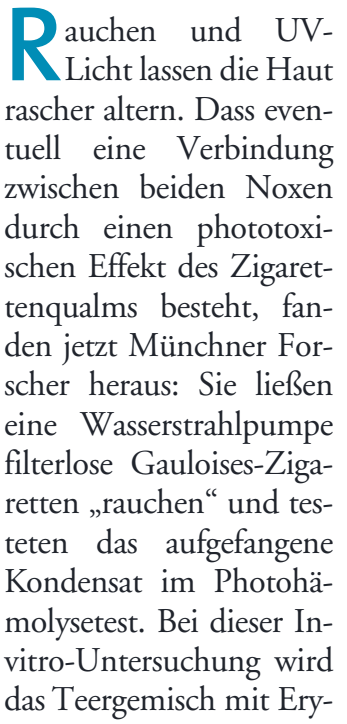

throzyten inkubiert, anschließend UVA- und UVB-Bestrahlung ausgesetzt und dann das Ausmaß der Zellzerstörung bestimmt. Vor allem die Kombination von UVA und Tabakkondensat setzte den Zellen zu - schon bei relativ geringer Energiezufuhr von $40 \mathrm{~J} / \mathrm{cm}^{2}$ waren $80 \%$ der Blutkörperchen zerplatzt. UVB brachte es "nur" auf 20\% hämolysierte Zellen. $\quad b k$

\section{Neurodermitis- Schulung wirkt!}

Ene deutsche Multicenter-Studie - konnte zeigen, dass strukturierte Neurodermitis-Schulungen eine signifikante Verbesserung von SCORAD und Lebensqualität in allen Altersgruppen bewirken - und zwar auch noch lange nach der Schulung. Geschult wurden bei bis zu 7-jährigen Kindern nur die Eltern, in der Gruppe der 8- bis 12Jährigen Eltern und Kinder zusammen und bei den 13-18-Jährigen die Betroffenen alleine. Die in der German Atopic Dermatitis Intervention Study validierte Schulung soll umgehend in den Versorgungsstrukturen umgesetzt werden, die Verhandlungen dazu laufen bereits. FK

Diepgen T. 20. Fortbildungskongress „Fortschritte der Allergologie, Immunologie und Dermatologie“, Davos, 1.-4. September 2004

\section{Ara $h 1$ ist abwaschbar}

들 rnussallergiker reagieren häufig - bereits auf kleinste Mengen Allergen mit schweren Symptomen und ergreifen deshalb teilweise skurril anmutende Vorsichtsmaßnahmen. Das ist eigentlich nicht nötig, scheint doch das Erdnuss-Hauptallergen Ara h 1 relativ einfach zu beseitigen, so zumindest das Ergebnis einer Studie: Normales Händewaschen mit gebräuchlichen Reinigungsmitteln reicht demnach aus, das Allergen praktisch vollständig zu eliminieren, bei Verwendung nur von Wasser bleiben allerdings vereinzelt nachweisbare Allergenmengen auf den Händen zurück. Auch Oberflächen von Tischen werden mit normalen Allzweckreinigern Erdnussallergen-frei. Und auch die Allergenbedrohung durch die Luft ist vernachlässigbar - weder offen herumstehende Erdnussbuttergläser noch das Essen von Erdnüssen belasten die Umgebung mit nennenswerten Mengen von Ara h 1.

Perry TT et al. J Allergy Clin Immunol 2004; 113: 973-6 